\title{
Computing 3D Non-rigid Brain Registration Using Extended Robust Point Matching for Composite Multisubject fMRI Analysis
}

\author{
Xenophon Papademetris ${ }^{3}$, Andrea P. Jackowski ${ }^{3}$, Robert T. Schultz ${ }^{3}$, \\ Lawrence H. Staib ${ }^{1,2}$, and James S. Duncan ${ }^{1,2}$ \\ 1 Departments of Elec. Engineering, ${ }^{2}$ Diag. Radiology, and ${ }^{3}$ Child Study Center, \\ Yale University New Haven, CT 06520-8042 \\ papad@noodle.med.yale.edu
}

\begin{abstract}
In this paper we present extensions to the Robust Point Matching framework to improve its ability to handle larger point sets with greater computational efficiency. While in the past this methodology has only been used to register either two-dimensional or small synthetic three-dimensional data-sets we demonstrate its first successful application on large real 3D data-sets. We apply this methodology to the problem of forming composite activation maps from functional magnetic resonance images. In particular we demonstrate the superior performance of this algorithm to a pure intensity-based registration in the specific area of the fusiform gyrus. We also demonstrate that the robustness of this method can be useful in the case where part of the brain is missing as a result of incorrect image slice specification.
\end{abstract}

\section{Introduction}

The nonrigid registration of the brain is a difficult task for which there are many important applications including comparing shape and function between individuals or groups, developing probabilistic models and atlases, measuring change within an individual and determining location with respect to a preacquired image during stereotactic surgery. The detailed comparison and nonrigid registration of brain images requires the determination of correspondence throughout the brain and the transformation of the image space according to these correspondences. In fMRI analysis, a key step is the formation of multisubject composite activation maps. Previously, Talairach-type methods [12] have been used but the limitations of these methods are well known. Nonrigid registration is necessary to bring the subjects into a common space.

There have been many approaches recently to nonrigid registration, with a particular emphasis on applications to brain imaging (see the collection [14]). The brain is of strong interest in neuroscience and neurosurgery and presents its own challenges. Most commonly, non-linear registration methods use image intensities to compute the transformation (e.g. $[1,6,13,9]$.) These techniques are potentially highly accurate but can be susceptible to local minima. In particular, 
the high anatomic variability of the cortex often results in intensity based methods yielding inaccurate results. Feature based methods have been developed to overcome such problems (e.g. [3,5,4].) None of these methods, however, is able to handle large variations in sulcal anatomy, as well as irregular sulcal branching and discontinuity.

Our proposed new method builds on and extents the robust point matching framework (RPM). RPM was originally presented in the context of joint estimation of rigid transformations (affine and piecewise-affine) and correspondences using softassign and deterministic annealing [8]. Developed in an optimization framework, this previous work models point matching as a linear assignmentleast squares problem. Softassign solves for fuzzy correspondences while enforcing the one-to-one constraint. Deterministic annealing gradually reduces the fuzziness of the correspondence in a controlled manner. Progressively refined transformation parameters are estimated by the resulting alternating update algorithm. Outliers are indirectly estimated using a robustness control term. The framework was extended to the non-linear registration case by Chui et al [2]. RPM has only been applied to the non-linear registration case [2] on simulated data and was hampered by (a) the use of a relatively small number of points and (b) the lack of outlier rejection, as a result of a clustering scheme used to increase the actual number of points.

In this paper, we present an extension to the robust point matching algorithm to address both of these issues. We explicitly use the measure of 'outlierness' estimated in the correspondence stage of this algorithm as a weight in the transformation estimation step to account for outliers in the template. Advanced numerical and graphics techniques such as sparse matrices and proper search strategies are also employed to enable the algorithm to use large numbers of points, which is important in the context of non-rigid brain registration. We evaluate our algorithm by testing its ability to form composites of functional magnetic resonance generated statistical parametric maps in the region of the fusiform gyrus. This region is especially important in the study of autism.

\section{Methods}

\subsection{A Review of Robust Point Matching}

We present here a slightly modified form of the standard RPM methodology as can be found in Chui and Rangarajan [2]. The registration procedure consists of two alternative steps: (i) the correspondence estimation step and (ii) the transformation estimation step. In the following discussion we will label the reference point set as $X$ and the transform point set as $Y$. The goal of the registration is to estimate the transformation $G: X \mapsto Y$. We will label $G^{k}$ the estimate of $G$ at the end of iteration $k . G^{0}$ is the starting transformation which can be the identity transformation.

Correspondence Estimation: Given the point sets $X$ and $Y$ we estimate the match matrix $M$, where $M_{i j}$ is the distance metric between points $G^{k}\left(X_{i}\right)$ and $Y_{j}$. The standard distance metric is defined as: 


$$
M_{i j}=\frac{1}{\sqrt{2 \pi T^{2}}} e^{\frac{-\left|G^{k}\left(X_{i}\right)-Y_{j}\right|^{2}}{2 T^{2}}}
$$

where $\left|X_{i}-Y_{j}\right|$ is the Euclidean distance between points $X_{i}$ and $Y_{j}$ and $T$ is the temperature term that controls the fuzziness of the correspondence. If the correspondence problem is to be thought of as a linear assignment problem, the rows and columns of $M$ must sum to 1 . The framework is further extended to handle outlier points by introducing an outlier column $C$ and an outlier row $R$. $C_{i}$ is a measure of the degree of 'outlierness' of a point in the reference point set $X_{i}$ and $R_{j}$ is the same for a point in the transform point set $Y_{j} . C$ and $R$ are initialized with constant values and then using an iterative procedure [8] the values of $M, C$ and $R$ are normalized such that:

$$
\forall i: \sum_{j} M_{i j}+C_{i}=1, \quad \text { and } \forall j: \sum_{i} M_{i j}+R_{j}=1
$$

Once the normalization is completed we can compute the correspondence as follows. Let $V_{i}$ be the corresponding point to $X_{i}$ and $w_{i}$ the confidence in the match. Then $V_{i}$ is defined as a normalized weighted sum of the points $Y_{j}$ where the weights are the elements of the match matrix $M$.

$$
V_{i}=\left(\sum_{j} M_{i j} Y_{j}\right) /\left(\sum_{j} M_{i j}\right), \quad \text { and } w_{i}=\left(\sum_{j} M_{i j}\right)=1-C_{i}
$$

Note that a point that has a high value in the outlier column $C$ will have low confidence and vice-versa.

Transformation Estimation: This is simply achieved by a regularized weighted least squares fit between $X_{i}$ and $V_{i}$ as follows:

$$
G^{k}=\underset{g}{\arg \min } \sum_{i} w_{i}\left(g\left(X_{i}\right)-V_{i}\right)^{2}+f(T) \mathcal{S}(g)
$$

where $\mathcal{S}(g)$ is a regularization functional (e.g. bending energy function) weighted by a function of the temperature $f(T)$. This last weighting term is used to decrease the regularization as we approach convergence.

Deterministic Annealing Framework: The alternating estimation of $M$ and $G$ is performed in a deterministic annealing framework. Starting with a high value of $T$ corresponding to a rough estimate of the maximum mis-alignment distance we first estimate $M$, and then $G$. Then $T$ is decreased by multiplying it with an annealing factor and the process is repeated until $T$ becomes sufficiently small.

\subsection{Extensions to RPM}

Extending the Correspondence to Use Labeled Points: In the original RPM framework all points are treated equally. However, we would like to ensure 
that a point originating on for example a given sulcus does not get mapped to a different sulcus. By adding labels to the points $X, Y$, we divide the points into groups and restrict correspondence accordingly. Using the label information we modify equation 1 to yield:

$$
M_{i j}=\delta\left(\mathbf{L}\left(\mathbf{X}_{\mathbf{i}}\right)-\mathbf{L}\left(\mathbf{Y}_{\mathbf{j}}\right)\right) \frac{1}{\sqrt{2 \pi T^{2}}} e^{\frac{-\left|G^{k}\left(X_{i}\right)-Y_{j}\right|^{2}}{2 T^{2}}}
$$

where $L\left(X_{i}\right)$ is the label of point $X_{i}$ and $\delta()$ is the delta function.

Improving Computational Efficiency: Previous uses of the RPM framework to estimate non-linear transformations have been limited by computational constraints. In particular the number of points used could no exceed about 200 which is clearly not adequate for estimating non-linear brain registrations. Using clustering techniques [2] the number of points could be as high as 2000, but this extension resulted in the loss of the ability to handle outliers. In this section we describe the key steps involved in making RPM a robust computationally efficient technique suitable for estimating non-rigid registrations using appropriately large numbers of points.

Correspondence Estimation: If the number of points to be used is large the size of the matrix $M$ gets prohibitively large. For example in a case where each point set consists of 10000 points storing $M$ requires $\approx 400 \mathrm{MB}$ of RAM. Further the algorithm will need to evaluate equation (4) $10^{8}$ times. By observation, however, only a small fraction of the elements of $M$ have significant values (rarely more than 2-3\%). Hence by using (i) a fast searching technique to identify only those points $Y_{i}$ that are closer to a point $X_{i}$ than a threshold (typically $<3 * T$ resulting in $M_{i j}>0.001$ ) and (ii) a sparse matrix representation for the matrix $M$ itself, we reduce the memory and computational requirements of the correspondence estimation from $O\left(p^{2}\right)$ to $O(p)$, where $p$ is the size of the point sets.

Transformation Estimation: We have selected a free-form deformation (FFD) model [9] to parameterize the transformation. The control points of the FFD model, unlike the thin-plate spline model [2], exert only a local influence on the transformation. Further, the points in the point set $X$ that are influenced by a given control point are the same during the estimation process and hence computing gradients as part of the minimization of equation 3 with respect to different control point locations can be achieved by only computing the fitting term for only those points in $X$ that are influenced by this change.

\section{Experimental Results}

We have tested our extended RPM-based registration algorithm in the challenging problem of forming composite activation maps from functional magnetic resonance data (fMRI). In particular we focus on visual processing that involve face recognition that activates the fusiform gyrus [10]. The fusiform is of particular importance in the study of autism. 
Data Acquisition and Pre-Processing: For a set of 10 normal controls, we acquired both anatomical (3D) images and fMRI data at $1.5 \mathrm{~T}$ using standard protocols in use at the Yale Child Study Center [10]. In particular the activations were derived using contrast from complex visual tasks. The activations were estimated using locally developed software and mapped to the three-dimensional anatomical images using a rigid-registration procedure [11]. For each subject we extracted: (i) the outer cortical surface using a quick approximate brain stripping procedure and sampled each outer surface to about 4000-4500 points and (ii) the bounding sulci of the left and fusiform gyri using manual delineation. By accurately delineating the fusiform, we can expect to achieve better registration of this functionally active area. There were about 300-400 points on each sulcus yielding a total point set size for each subject of 5500-6500 points (depending on brain size). The points were labeled to identify their surface of origin.

Registration Methodology: Composite activations were formed by registering the point sets using our proposed algorithm and a non-linear intensity based algorithm [9]. Both algorithms used the same FFD model for the transformation, hence the estimated transformations had approximately the same degrees of freedom. For the modified RPM algorithm, we use a starting temperature of $10 \mathrm{~mm}$ and progressively reduced this to about $1 \mathrm{~mm}$ by successive factors of 0.8. We first estimated an affine transformation (using the same framework) and then restarted the algorithm using the affine estimate as a starting point. The bspline-FFD transformation was set to have control points spaced at $15 \mathrm{~mm}$, which was approximately the same as for the intensity-based algorithm. ${ }^{1}$

Robustness Results: In this first example, we demonstrate the ability of the pointbased algorithm to deal with outliers. The original images are shown in figure 1(left-a) and (left-b). Note that the transform image is missing part of the head (at the edges) as the head volume did not fit in the standard slice specifications (the 3D images were acquired in a sagittal orientation.) The warped brain using the intensity based method is shown in figure $1($ left $-\mathrm{c})$. Note the significant distortion at both the extreme left and right portions of the brain. To perform the point based registration, we first construct a point model of the brain shown in figure 1(right-a). In the case of the second brain, the outer surface has a hole as a result of the missing structure, as shown in figure 1 (right-b). The corresponding portion of the brain surface from the reference brain is treated as an outlier by the algorithm and does not enter into the computation of the registration, resulting in a substantial reduction of the mis-registration artifact as shown in figure 1 (right-c).

fMRI Composite Results: The functional data from all subjects was processed by indvidual [10]. The statistical parametric maps (t-maps) from each subject were warped into a common space using the estimated transformations from the registration procedures and then averaged to form composite maps. A key

\footnotetext{
${ }^{1}$ Our implementation of this is based on the vtkCISG toolkit by Thomas Harkens (see http://image-registration.com.) We used standard parameters with the exception of the control point spacing.
} 


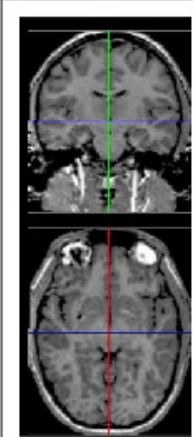

(a)

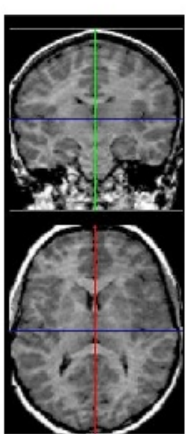

(b)

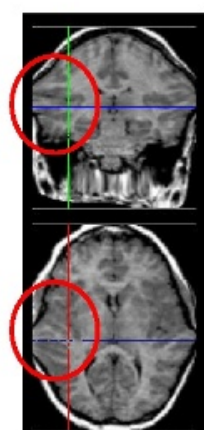

(c)

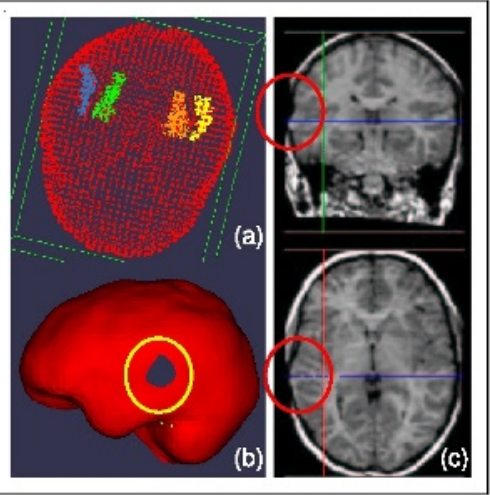

Fig. 1. Left: Registration artifacts with intensity-based method. (a) and (b) Reference and transform images respectively. Note that the transform image is missing part of the head close to the ears. (c) Registration result using intensity-based method. Note the large distortions (inside the red circles). Right: Robustness of Pointbased method. For the same subjects as figure 1.(a) Point-set used for point-based registration. (b) Close-up on outer surface of the transform image showing the hole in the outer surface. (c) point-based registration result (transform image warped to match reference image) showing absence of distortion.

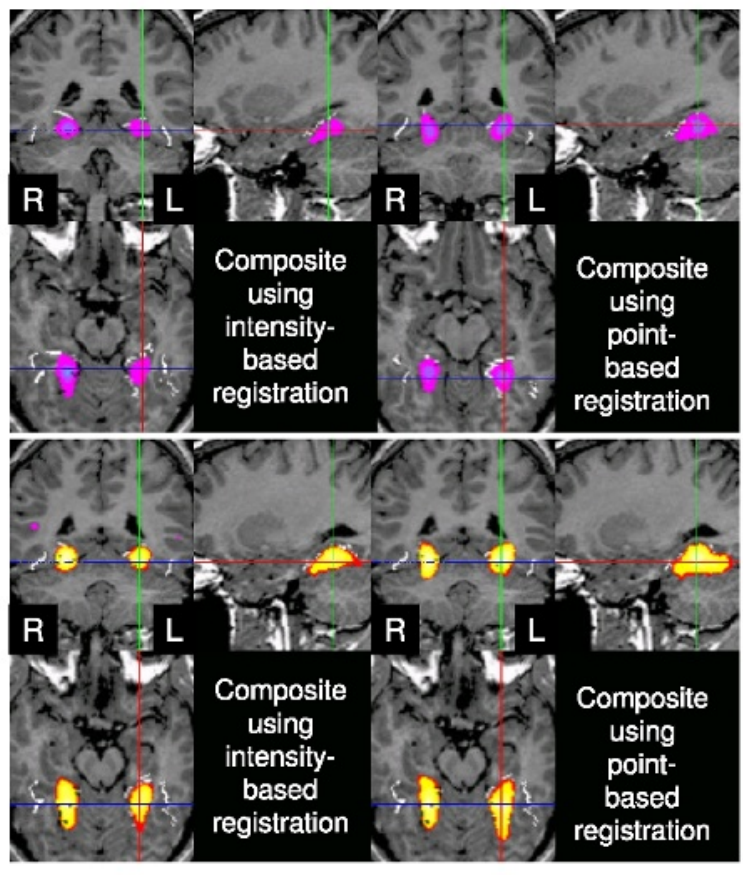

Fig. 2. Improved fMRI composite activations using point-based matching. (Top) Objects vs faces with different expressions and (Bottom) Faces vs Objects. The fusiform gyrus is activated as part of the recognition process yielding negative activations (top, purple-low to bluehigh) and positive activations (bottom red-low to yellowhigh). Note especially that the activations in the area of the left fusiform gyrus (shown on the right) are significantly bigger, reflecting better registration. 


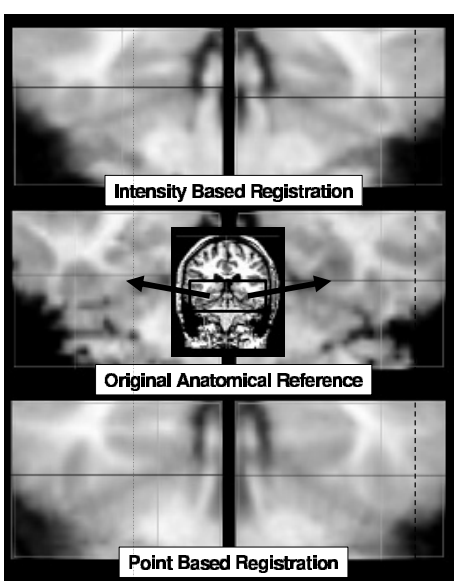

Fig. 3. Improved registration in the region of the fusiform. In the middle row we show single slice from the anatomical reference image with the area of the fusiform gyri zoomed in to the left and right. Top row : composite (average intensity) anatomical images produced using the intensity based method, and bottom row: composite anatomical images produced using our point-based method. Note the improved contrast in the composites produced by the point based method. In particular note that the white matter inside the fusifrom gyrus is completely blurred in the intensity-based composite whereas it is less blurred in the point-based composite.

limitation in using fMRI composites as an evaluation of registration accuracy is the generally low resolution of the fMRI data (in our case $3.125 \times 3.125 \times 9 \mathrm{~mm}$ ) compared to the anatomical data $(1.17 \times 1.17 \times 1.20 \mathrm{~mm})$. Further, unfortunately, in our case the fMRI data was pre-smoothed prior to the computation of the statistical parametric maps further reducing the effective resolution. Nonetheless, from the composites shown in figure 2, it can be seen that the point-based method demonstrates superior performance especially in the case of the left fusiform gyrus (shown in the right in the images). We assume that the method that yields larger regions of significant activations is more accurate as it more successfully pools data from different subjects.

We present results for two different composite activation maps. The maps created by both point-based and intensity based methods are identically thresholded. In particular note that in figure 2(top) the activation focus on the left fusiform has a core of higher value (blue) compared to the periphery (purple) whereas the intensity-based composite has only a smaller lower value (purple) activation area. The point based method also yields larger regions of significant activation for the second task shown in figure 2 (bottom), in the left fusiform gyrus. In addition, in figure 3 we show the results for the average anatomical images showing improved registration in the regions of the fusiform gyri.

\section{Conclusions}

We have demonstrated that a robust-point based technique can achieve improved fMRI composite activation maps as compared to more standard (and more automatic) intensity-based method. There are two general methods in use for generating multi-subject fMRI statistics. The general registration approach uses intensities only (e.g. SPM [7]) where anatomical variability such as in the shape of the fusiform gyrus is dealt with by blurring the images with a large Gaussian kernel. This blurring effect has the unwanted effect of reducing the effective resolution of the fMRI data. The alternative method, which is far more labor intensive, uses region of interest analysis (ROI) to outline regions of interest and compute statistics in these anatomically identified regions. Our method 
can be seen as a trade-off between the two approaches were the ROIs are used to influence the registration process, allowing voxelwise statistical measures.

In the future, we plan to combine the intensity-based and robust pointbased approaches. A key challenge is how to take advantage of the ability of the intensity-based methods to register sub-cortical structures without compromising the ability of the robust-point matching methods to reject outliers (caused by large anatomical differences) in the cortical regions.

Acknowledgments. The first author would like to acknowledge the help of Haili Chui.

\section{References}

1. G. E. Christensen, M. I. Miller, and M. W. Vannier. Individualizing neuroanatomical atlases using a massively parallel computer. Computer, 32-38, Jan. 1996.

2. H. Chui and A. Rangarajan. A new algorithm for non-rigid point matching. In Proceedings of IEEE Conf. on Computer Vision and Pattern Recognition-CVPR 2000, volume 2, pages 44-51. IEEE Press, 2000.

3. D. L. Collins, G. Le Goualher, and A. C. Evans. Non-linear cerebral registration with sulcal constraints. In Medical Image Computing and Computer Assisted Intervention, pages 974-984. Springer, Berlin, 1998.

4. I. Corouge, C. Barillot, P. Hellier, P. Toulouse, and B. Gibaud. Non-linear local registration of functional data. In Medical Image Computing and Computer Aided Intervention (MICCAI), pages 948-956, 2001.

5. C. Davatzikos. Spatial transformation and registration of brain images using elastically deformable models. Comp. Vision Image Under., 66(2):207-222, 1997.

6. J. Feldmar, G. Malandain, J. Declerck, and N. Ayache. Extension of the ICP algorithm to non-rigid intensity-based registration of 3D volumes. In Proc. Workshop Math. Meth. Biomed. Image Anal., pages 84-93, June 1996.

7. K. J. Friston, J. Ashburner, J. B. Poline, R. S. J. Frackowiak, and R. Turner. Spatial registration and normalization of images. Hum. Brain Mapping, 2:165189, 1995.

8. A. Rangarajan, H. Chui, and F. L. Bookstein. The softassign Procrustes matching algorithm. In Information Proc. Med. Imaging, pages 29-42, 1997.

9. D Rueckert, L. I. Sonoda, C. Hayes, D. L. G. Hill, M. O. Leach, and D. J. Hawkes. Non-rigid registration using free-form deformations: Application to breast MR images. IEEE Transactions on Medical Imaging, 18(8):712-721, August 1999.

10. R.T. Schultz, D.J. Grelotti, A. Klin, J. Kleinman, C. Van der Gaag, R. Marios, and P. Skudlarski. The role of the fusiform face area in social cognition: Implications for the pathobiology of autism. Phil. Trans. Roy. Soc, Ser. B, 458:415-427, 2003.

11. C. Studholme, D. Hill, and D. Hawkes. Automated 3D registration of magnetic resonance and positron emission tomography brain images by multiresolution optimisation of voxel similarity measures. Med. Phys., 24(1):25-35, 1997.

12. J. Talairach and P. Tournoux. Co-planar Stereotaxic Atlas of the Human Brain. Thieme Medical Publishers, New York, 1988.

13. J. P. Thirion. Image matching as a diffusion process: An analogy with maxwell's demons. Med. Image Anal., 2(3):243-260, 1998.

14. A. W. Toga. Brain Warping. Academic Press, San Diego, 1999. 